\title{
FAILURE CRITERION FOR BRICK MASONRY: A MICRO-MECHANICS APPROACH
}

\author{
MAREK KAWA \\ Wrocław University of Technology, Faculty of Civil Engineering, Wrocław, Poland, \\ e-mail: marek.kawa@pwr.edu.pl
}

\begin{abstract}
The paper deals with the formulation of failure criterion for an in-plane loaded masonry. Using micro-mechanics approach the strength estimation for masonry microstructure with constituents obeying the Drucker-Prager criterion is determined numerically. The procedure invokes lower bound analysis: for assumed stress fields constructed within masonry periodic cell critical load is obtained as a solution of constrained optimization problem. The analysis is carried out for many different loading conditions at different orientations of bed joints. The performance of the approach is verified against solutions obtained for corresponding layered and block microstructures, which provides the upper and lower strength bounds for masonry microstructure, respectively. Subsequently, a phenomenological anisotropic strength criterion for masonry microstructure is proposed. The criterion has a form of conjunction of Jaeger critical plane condition and Tsai-Wu criterion. The model proposed is identified based on the fitting of numerical results obtained from the microstructural analysis. Identified criterion is then verified against results obtained for different loading orientations. It appears that strength of masonry microstructure can be satisfactorily described by the criterion proposed.
\end{abstract}

Key words: strength anisotropy, micromechanics, masonry microstructure, masonry, failure criterion

\section{INTRODUCTION}

Brick masonry is one of the most traditional building materials. Although it has been known and used for centuries, its strength behavior is very difficult to describe. The microstructure of masonry: complex arrangement of bricks, bed and head joints effects in complex failure mechanisms and strength anisotropy of the material.

Within numerical analysis, engineering structures are usually modeled as a continuous medium with homogenized properties. For masonry microstructure, these properties are often obtained by employing finite element analysis at the level of periodic cell (see, for instance, works of Anthoine [1] and Shieh-Beygi and Pietruszczak [9]). The numerical implementation of such approach is however difficult and calculations require long computational time. A valid alternative, which allows focusing directly on failure condition, is to combine homogenization and the limit analysis.

Over last decades, homogenized limit analysis of masonry microstructure has been a subject of several studies. The upper bound strength assessment for microstructure consisting of infinitely resistant bricks and zero thickness joints has been presented in the work of De Buhan and De Felice [2]. The model has been subsequently implemented as macroscopic plasticity function into FE code for analysis of ancient masonry (De Felice et al. [3]). Precise lower bound assessment of critical load for masonry microstructure has been proposed by Milani et al. [6]. In the cited work, stress fields in few rectangular segments of masonry periodic cell have been described by the $n$-th degree polynomials. The values of the fields have been then optimized in the selected nodes of each segment subjected to the equilibrium, continuity of the stress vector and the plastic admissibility conditions. Macroscopic strength criterion obtained for fields described with polynomial of 3rd degree has been then implemented to the numerical code. The obtained macroscopic strength assessment in the above-mentioned cases has been given in space of principal stresses in a form of set of intersecting planes.

The focus of the present work is to propose macroscopic strength criterion for masonry microstructure under in-plane loading. The proposed criterion has a form of conjunction of Jaeger [4] and Tsai-Wu [11] model. These two parts have been associated with different failure mechanisms: critical plane cri- 
terion has been associated with localized shearing along the bed joints and Tsai-Wu criterion with others, more complicated mechanisms involving failure in both bed joints and head joints. The complete criterion is identified based on fitting in numerical results of lower bound strength assessment of masonry obtained with micro-mechanics approach and subsequently verified against results obtained for different load paths.

The present study is a preliminary one. The micromechanics approach employed to assess strength of masonry, which has been previously developed by the author (Kawa et al. [5]) is a simple one. The respective micro-stress fields constructed within periodic cell of masonry, which represent variables in formulated constrained optimization problem, can vary only linearly in selected segments of the cell. This makes the assessment results obtained, for some loading paths, not accurate. Verification of performance of the approach against numerical solution obtained for block and layered microstructure shows that for a number of other loading paths the procedure utilized provides good estimate of masonry strength.

\section{HOMOGENIZATION TECHNIQUE}

In the framework of micromechanics, macroscopic strength criterion represents a set of admissible macro stress states. The macro stress state is admissible if associated micro stress field is selfequilibrated and does not violate the respective failure criterion for all constituents involved. In addition, for periodic structures, the micro stress field has to be locally periodic. The above formulation can be written as (Suquet [10])

$$
\begin{gathered}
f\left(\sigma_{i j}\right) \leq 0 \Leftrightarrow\left\{\sigma_{i j}\right\} \in A \\
=\left\{\left\langle\tau_{i j}\right\rangle \mid \exists \tau_{i j}(y): \frac{\partial \tau_{i j}(y)}{\partial y}\right. \\
\left.=0, f\left(\tau_{i j}(y), y\right) \leq 0, \tau_{i j}(y)-\text { periodic }\right\},
\end{gathered}
$$

where $\sigma_{i j}, \tau_{i j}$ represent macro stress tensor and associated micro stress field, respectively, $f\left(\tau_{i j}(y), y\right)$ is a local strength criterion at a point with coordinates $y \in V_{\mathrm{RVE}}$, and $V_{\mathrm{RVE}}$ is a volume of a periodic cell. The symbol <.> denotes operation of volume averaging over a periodic cell.

The above definition (1), by virtue of limit theorems, provides a lower bound assessment of microstructure strength. For given loading conditions the value of the assessment can be specified by solving constrained optimization problem. The variables of the problem are the values of micro stress fields at every point of periodic cell, while the constraints are represented by the equilibrium, boundary and local plastic admissibility conditions. In order to improve numerical efficiency of the problem the number of variables is usually limited by assuming some idealized micro stress fields. The quality of obtained assessment depends on how well the assumed micro stress fields approximate the actual ones.

The geometry of periodic cell of the structure considered is presented in Fig. 1. Given how the cell repeats itself within the panel, "transition" of periodicity along horizontal boundaries is required. The traction is then equal and opposite in pairs (Fig. 1): a and d, $\mathrm{b}$ and $\mathrm{e}$, as well as $\mathrm{f}$ and $\mathrm{c}$.

The methodology of construction of simple stress fields within a given periodic cell of masonry microstructure have been presented in detail in earlier work

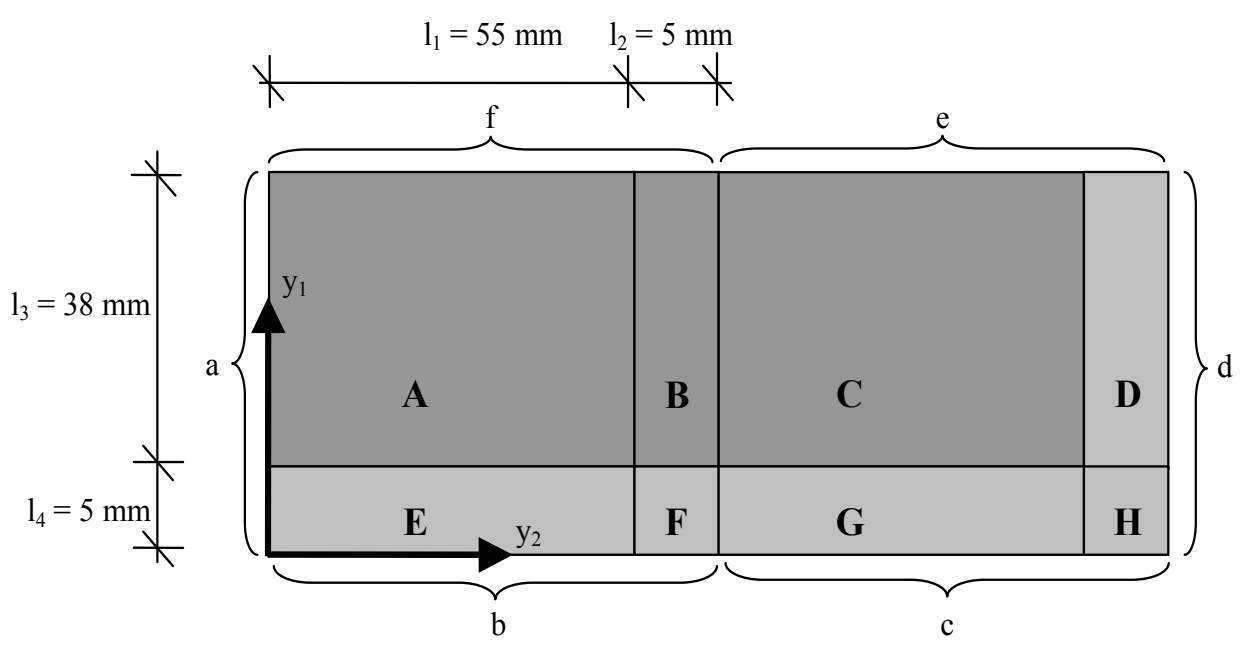

Fig. 1. Assumed geometry of periodic cell of masonry 
of the author (Kawa et al. [5]). In the cited work it has been shown that the assumed micro stress fields correspond to typical failure mechanisms for masonry. Based on those fields constrained optimization problem has been formulated. In order to introduce the methodology in the present study the basic steps of this formulation are presented:

(i) The plain stress state is assumed. The micro stress fields are constructed as follows: The representative cell is divided into 8 segments (Fig. 1), each made of brick or mortar. The fields are assumed to be constant in segments B, D, E and G. In segments $A, C, F$ and $H$ the values of the fields can vary linearly from one boundary of the segment to the other. Vertical normal micro stress $\tau_{11}$ varies only vertically, horizontal normal micro stress $\tau_{22}$ - only horizontally and micro shear stress $\tau_{12}$ in both directions. Since only liner variation of the fields is possible the local equilibrium conditions are satisfied at every point of periodic cell, if the global equilibrium conditions are satisfied, namely,

$$
\begin{gathered}
\left(\tau_{22}^{B}-\tau_{22}^{D}\right) \cdot l_{3}=2 \tau_{12}^{E G} \cdot l_{1} ; \quad\left(\tau_{22}^{E}-\tau_{22}^{G}\right) \cdot l_{4}=2 \tau_{12}^{B D} \cdot l_{2} ; \\
\left(\tau_{11}^{B}-\tau_{1}^{D}\right) \cdot l_{2}=\tau_{12}^{E G} \cdot l_{4} ; \quad\left(\tau_{11}^{E}-\tau_{11}^{G}\right) \cdot l_{1}=\tau_{12}^{B D} \cdot l_{3},
\end{gathered}
$$

where $\tau_{11}^{K}, \tau_{22}^{K}$ denote normal micro stresses in segment $\mathrm{K}$ and $\tau_{12}^{B D}, \tau_{12}^{E G}$ are local correctors (with average equal to 0 ) of the macro shear stress which are equal and opposite in pairs of the segments $\mathrm{B}$ and $\mathrm{D}$ as well as $\mathrm{E}$ and $\mathrm{G}$, respectively. The assumed stress fields satisfy periodic boundary conditions with transition.

(ii) The macro stress tensor is defined in terms of its principal values and angle $\alpha$ which orients base vectors with respect to head joints. The consistency conditions between micro and macro stress fields can be expressed as

$$
\begin{gathered}
\left(\tau_{11}^{B} l_{2}+\tau_{11}^{D} l_{2}+\tau_{11}^{E} l_{1}+\tau_{11}^{G} l_{1}\right) /\left(2 l_{1}+2 l_{2}\right) \\
=\sigma_{1} \cos ^{2} \alpha+\sigma_{2} \sin ^{2} \alpha, \\
\left(\tau_{22}^{B} l_{3}+\tau_{22}^{D} l_{3}+\tau_{11}^{E} l_{4}+\tau_{11}^{G} l_{4}\right) /\left(2 l_{3}+2 l_{4}\right) \\
=\sigma_{1} \sin ^{2} \alpha+\sigma_{2} \cos ^{2} \alpha, \\
\sigma_{12}=\left(\sigma_{2}-\sigma_{1}\right) \cos \alpha \sin \alpha,
\end{gathered}
$$

where $l_{i}$ are geometry parameters (Fig. 1) and $\sigma_{12}$ is the average value of shear stress over the periodic cell. (iii) When the assumed field is constructed according to (i) and (ii) the plastic admissibility needs to be checked only at 12 points of periodic cell, which are:

- any point within segments B, D, E and G; the micro stress field values in these segments are

$$
\begin{array}{ll}
\tau_{i j}^{B}=\left\{\tau_{11}^{B}, \tau_{22}^{B}, \sigma_{12}+\tau_{12}^{B D}\right\}, & \tau_{i j}^{D}=\left\{\tau_{11}^{D}, \tau_{22}^{D}, \sigma_{12}-\tau_{12}^{B D}\right\}, \\
\tau_{i j}^{E}=\left\{\tau_{11}^{E}, \tau_{22}^{E}, \sigma_{12}+\tau_{12}^{E G}\right\}, & \tau_{i j}^{G}=\left\{\tau_{11}^{G}, \tau_{22}^{G}, \sigma_{12}-\tau_{12}^{E G}\right\},
\end{array}
$$

- all corners of segment $\mathrm{A}$ or $\mathrm{C}$; the micro stress field values in these corners are

$$
\begin{aligned}
& \tau_{i j}^{A t l}=\tau_{i j}^{C b r}=\left\{\tau_{11}^{G}, \tau_{22}^{D}, \sigma_{12}-\tau_{12}^{B D}-\tau_{12}^{E G}\right\}, \\
& \tau_{i j}^{A t r}=\tau_{i j}^{C b l}=\left\{\tau_{11}^{G}, \tau_{22}^{B}, \sigma_{12}+\tau_{12}^{B D}-\tau_{12}^{E G}\right\}, \\
& \tau_{i j}^{A b l}=\tau_{i j}^{C t r}=\left\{\tau_{11}^{E}, \tau_{22}^{D}, \sigma_{12}-\tau_{12}^{B D}+\tau_{12}^{E G}\right\}, \\
& \tau_{i j}^{A t r}=\tau_{i j}^{C b l}=\left\{\tau_{11}^{E}, \tau_{22}^{B}, \sigma_{12}+\tau_{12}^{B D}+\tau_{12}^{E G}\right\},
\end{aligned}
$$

where $\tau_{i j}^{A t l}$ denotes micro stress fields values in "top left" corner of segment A, etc.

- all corners of segment $\mathrm{F}$ or $\mathrm{H}$; the micro stress field values in these corners are

$$
\begin{aligned}
& \tau_{i j}^{F t l}=\tau_{i j}^{H b r}=\left\{\tau_{11}^{B}, \tau_{22}^{E}, \sigma_{12}+\tau_{12}^{B D}+\tau_{12}^{E G}\right\}, \\
& \tau_{i j}^{F t r}=\tau_{i j}^{H b l}=\left\{\tau_{11}^{B}, \tau_{22}^{G}, \sigma_{12}+\tau_{12}^{B D}-\tau_{12}^{E G}\right\}, \\
& \tau_{i j}^{F b l}=\tau_{i j}^{C t r}=\left\{\tau_{11}^{D}, \tau_{22}^{E}, \sigma_{12}-\tau_{12}^{B D}-\tau_{12}^{E G}\right\}, \\
& \tau_{i j}^{A b r}=\tau_{i j}^{C t l}=\left\{\tau_{11}^{D}, \tau_{22}^{G}, \sigma_{12}-\tau_{12}^{B D}-\tau_{12}^{E G}\right\} .
\end{aligned}
$$

The parameters of strength criterion that need to be checked vary depending on the constituent which forms the segment considered.

(iv) The equilibrium equations (2), consistency between micro/macro fields (3), and plastic admissibility conditions at the 12 points of periodic cell form a complete set of constrains of the optimization problem considered. The objective of optimization is to maximize or minimize one of the principal stresses (e.g., $\sigma_{1}$ ) for given values of the other principal stress (e.g., $\sigma_{2}$ ) and orientation angle $\alpha$. The solution has been obtained in AMPL environment (www.ampl.com) using the IPOPT solver. IPOPT uses primaldual interior point method in order to solve 

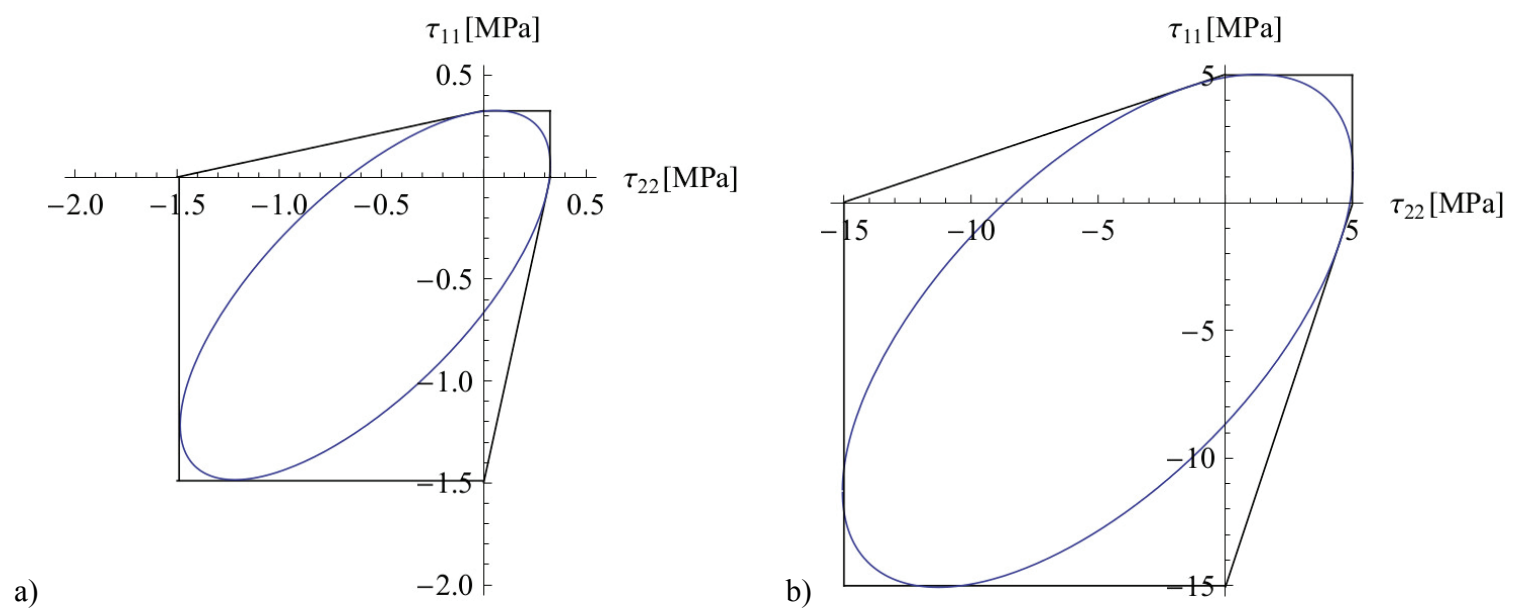

Fig. 2. The Mohr-Coulomb criterion with inscribed Drucker-Prager criterion for mortar (interface) (a) and brick (b)

nonlinear constrained optimization problem. By solving a number of optimization problems, both directional strength test results and admissible macro stress filed in $\sigma_{1}-\sigma_{2}$ space can be obtained. Since the assumed micro stress filed is idealized these results cannot be treated as exact solution - they provide a lower bound of masonry strength.

Local criterion of failure for both constituents of masonry, i.e., brick and mortar has been assumed as the Drucker-Prager one. Under plane stress conditions, when $x_{3}$ is chosen as out-of-plane direction, the criterion can be expressed as

$$
f\left(\tau_{i j}\right)=a\left(\tau_{11}+\tau_{22}\right)
$$

$+\sqrt{\frac{1}{6}\left(\left(\tau_{11}-\tau_{22}\right)^{2}+\left(\tau_{11}\right)^{2}+\left(\tau_{22}\right)^{2}\right)+\left(\tau_{12}\right)^{2}}-k \leq 0$.

Parameters of the Drucker-Prager criterion for the components of the microstructure have been assumed in such a way that the criterion inscribes (Fig. 2) the Mohr-Coulomb condition with its typical parameters for masonry constituents used in earlier work (Kawa et al. [5]). Typical Mohr-Coulomb and associated Drucker-Prager parameters of the constituents, which are related through equations

$$
k=\sqrt{3} c \sqrt{\frac{1}{3+4 \tan ^{2} \phi}}, \quad a=\frac{\tan \phi}{\sqrt{9+12 \tan ^{2} \phi}},
$$

are summarized in Table 1.

The assumption of Drucker-Prager criterion for description of strength of the masonry constituents may raise some doubts. It is well-known that for typical parameters, both the Mohr-Coulomb and Drucker-Prager criterion strongly overestimate strength of masonry in tension. This is mainly the effect of brick-mortar interface having very low tensile resistance. Usually, to fit in the experimental results additional tension Cut-Off criterion is used which limits the mortar strength in tension zone. In the present paper, the mechanism of tensile failure of brick-mortar interface has not been considered. The author focuses on the influence of assumed local strength criterion describing shearing and compressive resistance of constituents on the shape of macroscopic failure criterion for masonry microstructure. For this purpose, the assumption of pure DruckerPrager criterion as governing constituents strength seems to be justified. One should note, however, that the obtained results of microstructure strength in tensile zone can be inconsistent with real masonry strength.

Table 1

The Mohr-Coulomb and associated Drucker-Prager parameters for masonry constituents

\begin{tabular}{|c|c|c|c|c|}
\hline & \multicolumn{2}{|c|}{ Mohr-Coulomb } & \multicolumn{2}{c|}{ Drucker-Prager } \\
\hline Constituent & $c[\mathrm{MPa}]$ & $\alpha$ & $k[\mathrm{MPa}]$ & $a$ \\
\hline Brick & 4.35 & $30^{\circ}$ & 3.61 & 0.16 \\
\hline Mortar & 0.35 & $40^{\circ}$ & 0.25 & 0.20 \\
\hline
\end{tabular}

In Figs. 3 and 4, the numerical results obtained for simulated directional uniaxial compression and tension tests are presented. Strong dependence of masonry strength on test direction can be observed in both figures.

For specified load directions $\alpha$, also the lower bound of admissible macro stress filed has been obtained numerically. The results obtained for $\alpha=0$ and $\alpha=22.5^{\circ}$ are shown in Figs. 5 and 6 . As can be observed in the figures, the shape of the assessment strongly depends on angle $\alpha$. 

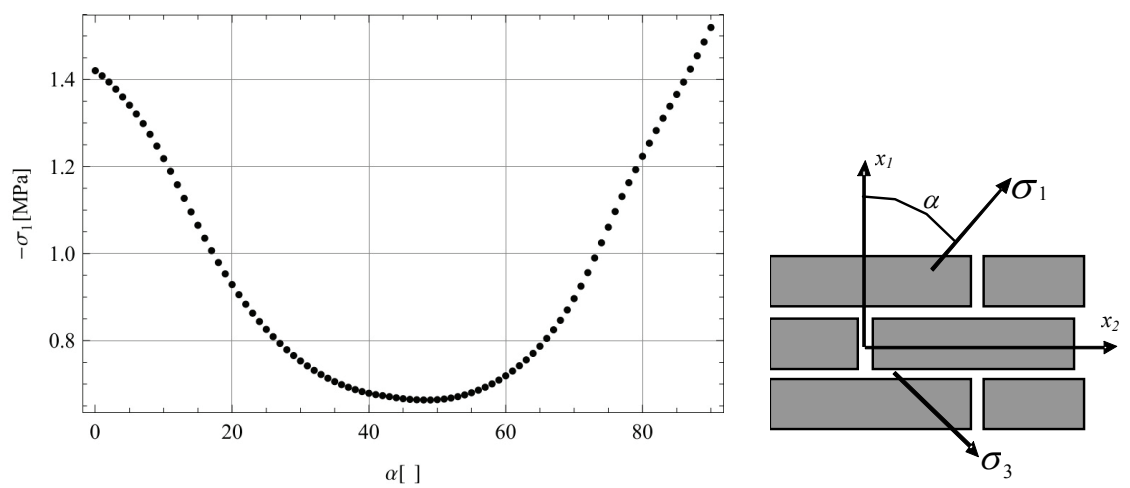

Fig. 3. Lower bound strength assessment for masonry. Directional uniaxial compression
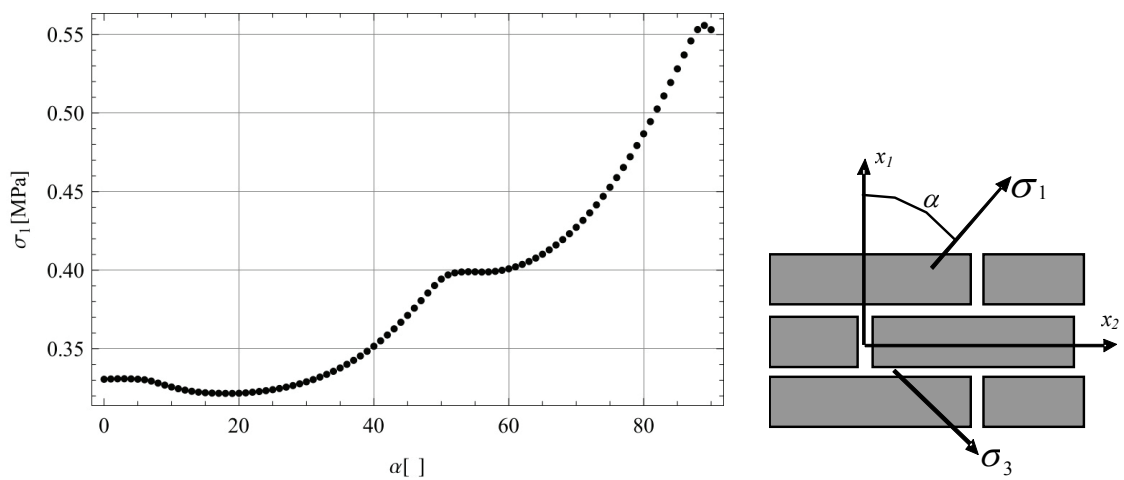

Fig. 4. Lower bound strength assessment for masonry. Directional uniaxial tension test
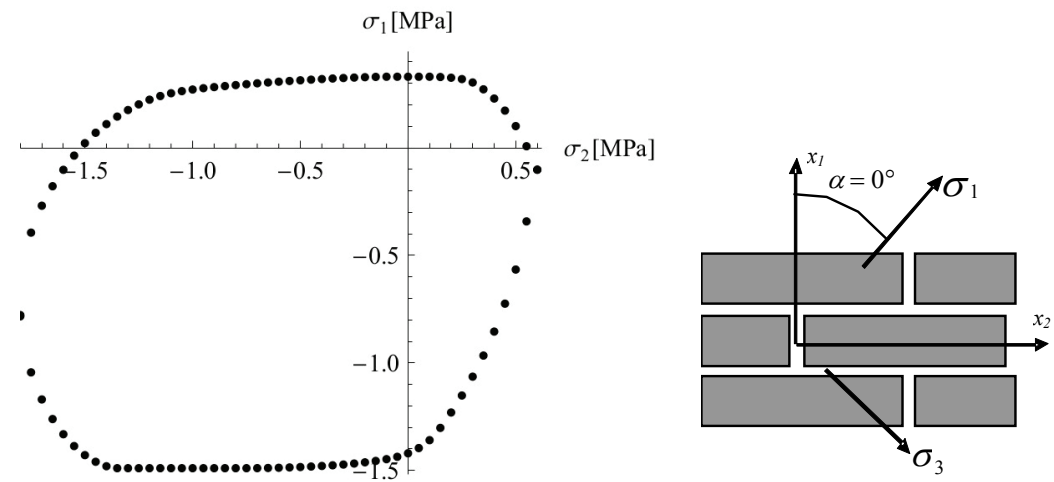

Fig. 5. Assessment of macroscopic failure criteria for masonry in principal stress space for load angle $\alpha=0^{\circ}$
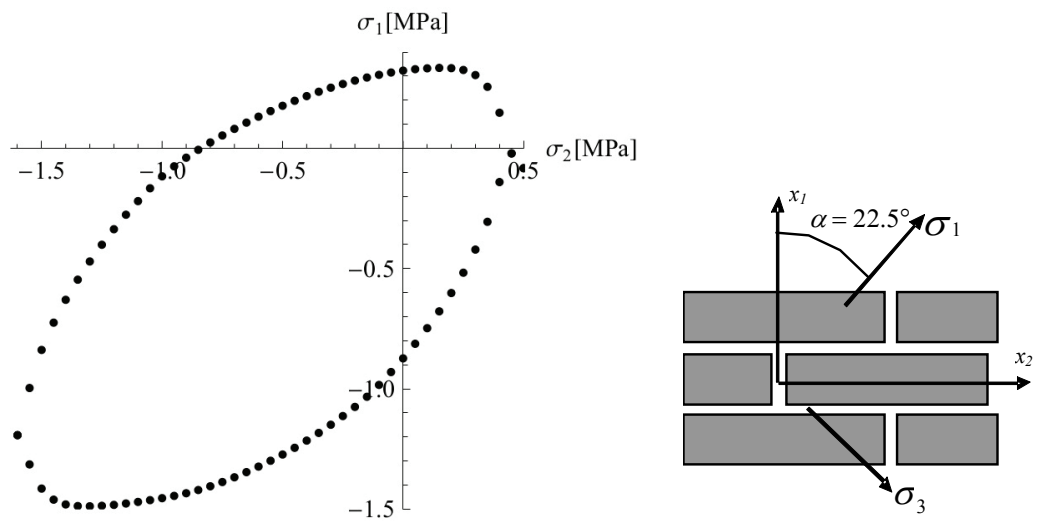

Fig. 6. Assessment of macroscopic failure criteria for masonry in principal stress space for load angle $\alpha=22.5^{\circ}$ 


\section{LAYERED AND BLOCK MICROSTRUCTURES}

It is easy to notice that with replacement of the material in selected segments of its periodic cell, masonry microstructure can be converted to either layered or block microstructure (Fig. 7). Both of these microstructures are much simpler than masonry microstructure and the micro stress fields for them are much easier to construct. For layered microstructure only two segments of periodic cell can be distinguished (Fig. 8). Moreover, two of the micro stress components $\tau_{11}$ and $\tau_{12}$ are constant in the periodic cell and thus equal to its macroscopic values $\sigma_{11}$ and $\sigma_{12}$. The only unknown components of micro stress tensor are values of $\tau_{22}$ in two constituents of microstructure. The constrains of optimization problem presented in the previous section for masonry microstructure can be reduced to three consistency conditions

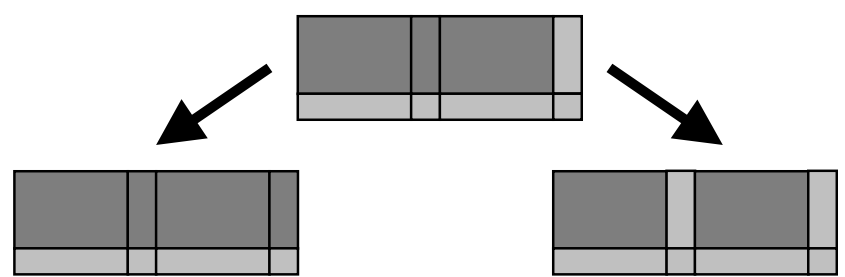

Fig. 7. Transformations of masonry microstructure to layered and block microstructures

$$
\begin{gathered}
\sigma_{1}=\sigma_{1} \cos ^{2} \alpha+\sigma_{2} \sin ^{2} \alpha \\
\left(\tau_{22}^{A} l_{3}+\tau_{22}^{B} l_{4}\right) /\left(l_{3}+l_{4}\right)=\sigma_{1} \sin ^{2} \alpha+\sigma_{2} \cos ^{2} \alpha \\
\sigma_{12}=\left(\sigma_{2}-\sigma_{1}\right) \cos \alpha \sin \alpha
\end{gathered}
$$

and plastic admissibility conditions for points in two segments of microstructure. The micro stress field values at these points are: $\tau_{i j}^{A}=\left\{\sigma_{11}, \tau_{22}^{A}, \sigma_{12}\right\}$, $\tau_{i j}^{P}=\left\{\sigma_{11}, \tau_{22}^{B}, \sigma_{12}\right\}$.

For block microstructure the number of segments rises to four (Fig. 9). The shear component of micro stress filed $\tau_{12}$ is also constant in periodic cell and equal to macroscopic stress value $\sigma_{12}$. The unknown values of micro stress fields are $\tau_{11}$ and $\tau_{22}$ which are constant in the vertical (A-C, B-D) and horizontal (A-B, C-D) stripes of segments, respectively. The reduced set of constrains of optimization problem is formed by consistency conditions

$$
\begin{gathered}
\left(\tau_{11}^{A} l_{1}+\tau_{11}^{B} l_{2}\right) /\left(l_{1}+l_{2}\right)=\sigma_{1} \cos ^{2} \alpha+\sigma_{2} \sin ^{2} \alpha \\
\left(\tau_{22}^{A} l_{3}+\tau_{22}^{C} l_{4}\right) /\left(l_{3}+l_{4}\right)=\sigma_{1} \sin ^{2} \alpha+\sigma_{2} \cos ^{2} \alpha \\
\sigma_{12}=\left(\sigma_{2}-\sigma_{1}\right) \cos \alpha \sin \alpha
\end{gathered}
$$

and plastic admissibility conditions for points in four segments of microstructure with micro stress fields values: $\tau_{i j}^{A}=\left\{\tau_{11}^{A}, \tau_{22}^{A}, \sigma_{12}\right\}, \tau_{i j}^{B}=\left\{\tau_{11}^{B}, \tau_{22}^{A}, \sigma_{12}\right\}, \quad \tau_{i j}^{C}=$ $\left\{\tau_{11}^{A}, \tau_{22}^{C}, \sigma_{12}\right\}, \tau_{i j}^{D}=\left\{\tau_{11}^{B}, \tau_{22}^{C}, \sigma_{12}\right\}$.

For both layered and block microstructures the presented distributions of micro stress actually appear in the periodic cell at the beginning of failure. The results obtained by solving constrained optimization problems identically as for masonry microstructure for these two microstructures can be regarded as exact description of their strength. It is evident that if identical strength criteria for constituents are assumed the layered microstructure (no head joints) is stronger, and block microstructure (continuous head joints) is weaker than corresponding masonry microstructure. The numerical solutions obtained for these microstructures can be used as upper and lower assessment of masonry strength, respectively.

In Figs. 10 and 11, the numerical solutions for layered and block microstructures are presented together with previously obtained numerical results for masonry.

The graphs presented confirm the fact that results obtained for masonry microstructure are bounded by strength of block and layered microstructure. Since the strength function for masonry (dotted line) presented in the diagram represents lower bound assessment of masonry strength, the exact solution of the problem lies between the dotted and the black line. It can be seen in both diagrams that for a wide range of angles ( 0 to $45^{\circ}$ for tension and 0 to $70^{\circ}$ for compression) the results obtained for masonry almost coincide with numerical solution for layered microstructure. The analysis of results obtained for layered microstructure indicates that, for a wide range of load orientations (including the range mentioned above), the strength of the material is associated with failure of its weaker constituent only. This mechanism of failure, which usually involves localized shearing along lamination plane, has been reported by Jaeger [4]. Thus, when results obtained for masonry and layered microstructure coincide, identical mechanism of failure, i.e., localized shearing along bed joints, most likely occurs also in masonry microstructure. The analysis of values of microstress fields obtained for masonry microstructure confirms this hypothesis. 

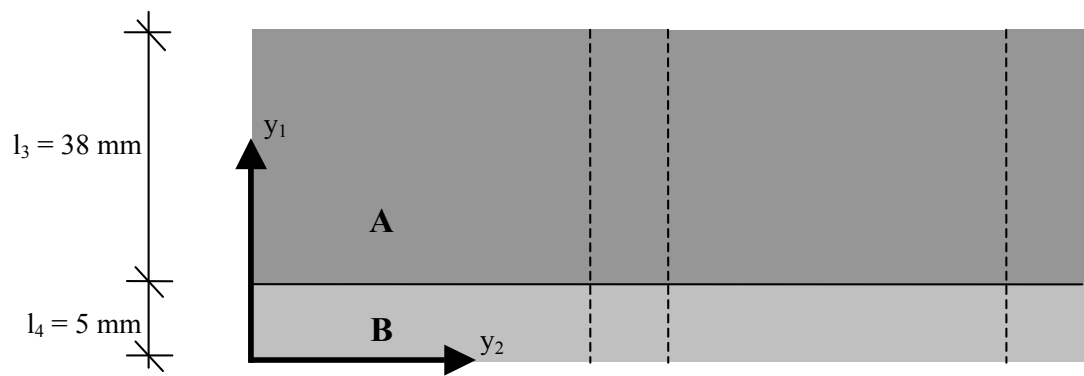

Fig. 8. Geometry of layered microstructure considered

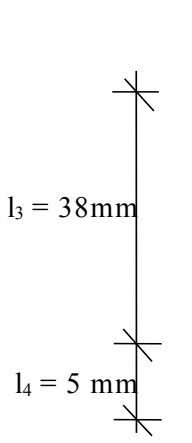

$$
1_{1}=55 \mathrm{~mm} \quad 1_{2}=5 \mathrm{~mm}
$$

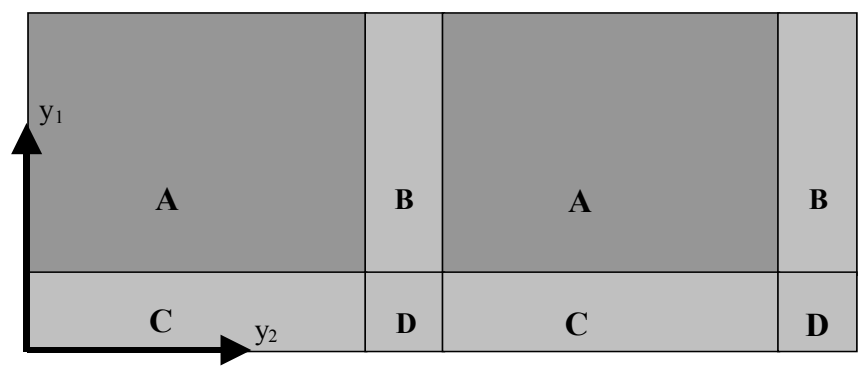

Fig. 9. Geometry of block microstructure considered
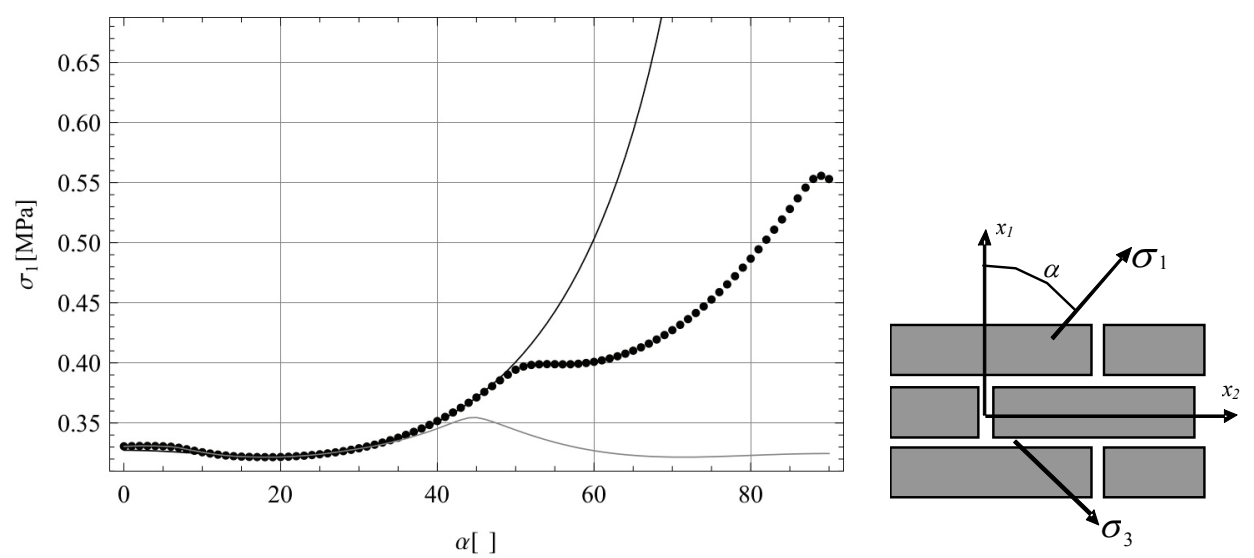

Fig. 10. Lower bound strength assessment for masonry (dots) and exact strength values for layered (black line) and block (gray line) microstructures. Directional uniaxial tension test
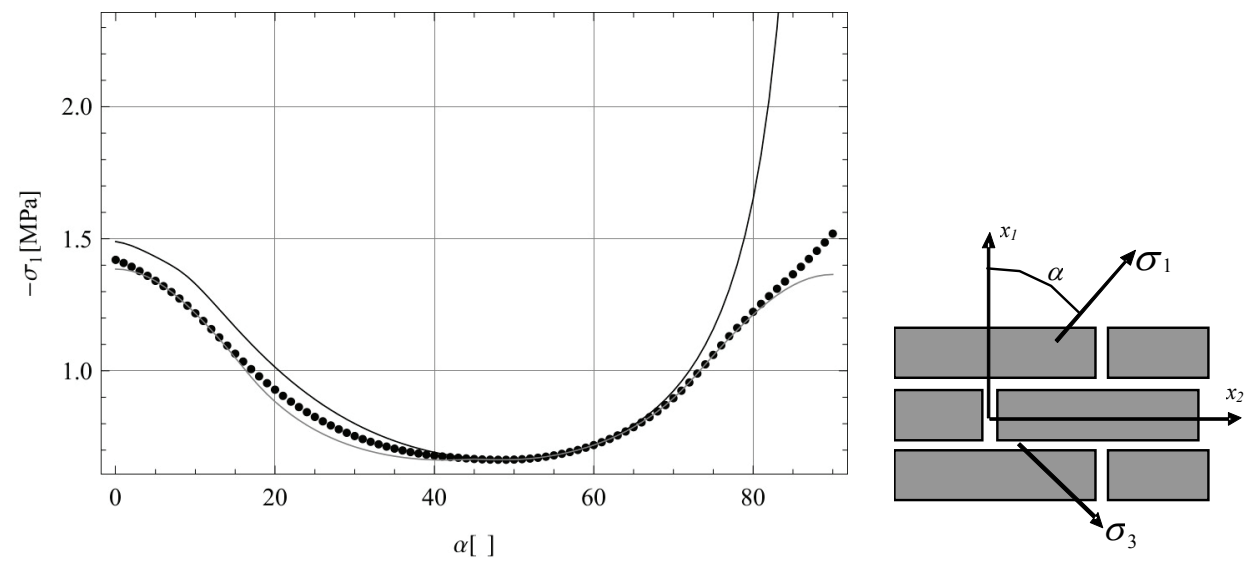

Fig. 11. Lower bound strength assessment for masonry (dots) and exact strength values for layered (black line) and block (gray line) microstructures. Directional uniaxial tension test 


\section{PROPOSED FAILURE CRITERION: IDENTIFICATION AND VERIFICATION}

As can be seen from both numerical simulations (Shieh-Beygi and Pietruszczak [9]) and experimental studies (Page [8]), destruction of brick masonry in the majority of cases occurs due to failure of its weaker component, i.e., mortar (interface). The failure can occur either only in bed joint, in head joint and a part of bed joint (zigzag pattern) or in all joints simultaneously. The two latter mechanisms are quite complicated: selected cases were analyzed in the earlier work (Kawa et al. [5]). As mentioned in the previous paragraph, simple mechanism of failure in bed joints only is virtually identical to mechanism of localized shearing, reported for sedimentary rocks by Jaeger [4].

In the work by Jaeger [4], a simple anisotropic criterion has been presented which distinguished two main mechanisms of failure for sedimentary rock. The model of material consists of an isotropic matrix and inclusions in the form of identically oriented, dimensionless weakness planes. Failure of material occurs either when matrix reaches its strength or when vector of load on weakness plane causes localized shearing. Strength criterion for the model is then a conjunction of an isotropic criterion for the matrix and a criterion for weakness plane expressed in terms of the stress vector.

The identically oriented bed joints in a masonry microstructure can also be interpreted as weakness planes. Following the idea of Jaeger [4] macroscopic strength condition for masonry should consist of weakness plane condition and some other criterion for matrix. If direction $x_{1}$ is assumed as perpendicular to the bed joints, the weakness plane criterion for the Drucker-Prager material, under plane stress conditions, can be expressed as

$$
\begin{gathered}
f_{c}\left(\sigma_{i j}\right)=\sqrt{\sigma_{12}^{2}} \\
-\frac{1}{2} \sqrt{\frac{4 k_{c}^{2}-12 a_{c} k_{c} \sigma_{11}-\left(1-12 a_{c}^{2}\right) \sigma_{11}^{2}}{1-3 a_{c}^{2}}} \leq 0 .
\end{gathered}
$$

In the above formula, $\sigma_{11}$ and $\sigma_{12}$ are appropriate components of stress vector on weakness plane and $a_{c}$,

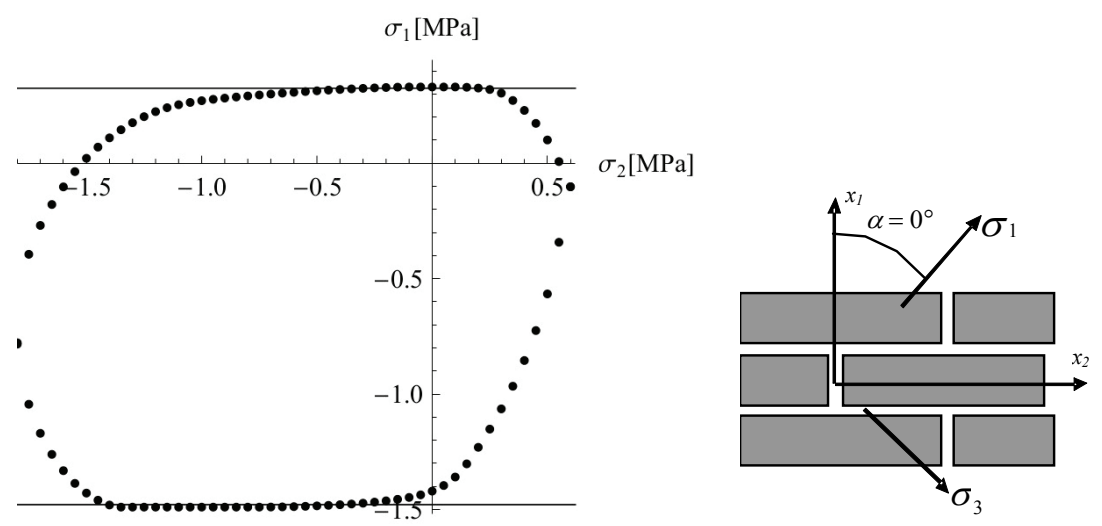

Fig. 12. Critical plane condition (line) together with numerical results for masonry. Load angle $\alpha=0^{\circ}$
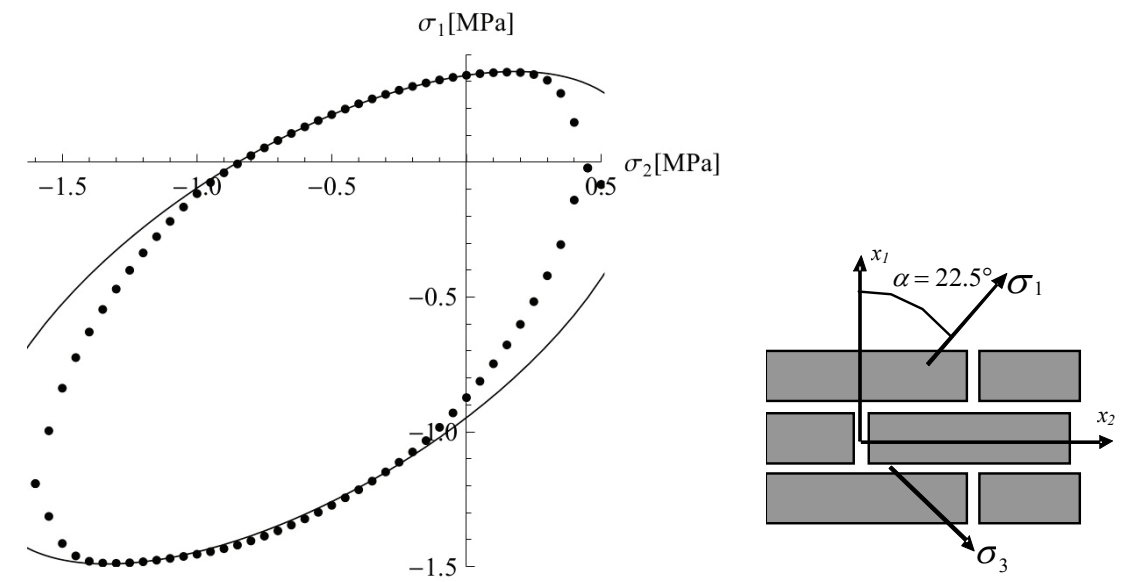

Fig. 13. Critical plane condition (line) together with numerical results for masonry. Load angle $\alpha=22.5^{\circ}$ 
$k_{c}$ are the Drucker-Prager parameters for weakness plane, which for the case considered should be assumed identical as those of mortar.

Function $f_{c}\left(\sigma_{i j}\right)=0$, transformed to principal stress space is presented in Figs. 12 and 13 for two different load directions $\left(\alpha=0, \alpha=22.5^{\circ}\right)$ together with assessment of strength criterion for masonry obtained in Section 2. As can be seen for some load paths the strength of masonry microstructure coincides with critical plane criterion.

The results presented in Figs. 12 and 13, which do not coincide with weakness plane criterion, are obviously associated with some other mechanism of masonry failure, different than localized shearing along bed joints. In the model proposed by Jaeger [4] the strength for cases not associated with failure along weakness plane are typically represented by an isotropic criterion of a matrix. Observing Figs. 12 and 13 it can be clearly seen that for these parts of plot in which the results do not coincide with the weakness plane criterion the strength is not the isotropic one. Natural extension of Jaeger model is to replace an isotropic criterion for a matrix with the properly defined anisotropic one. This idea has been used in one of the previous works of the author (Lydzba and Kawa [7]) to describe strength of layered microstructure. In the cited work, for Drucker-Prager components, the Pariseau criterion has been chosen as the most appropriate one for matrix. Here, for the case of brick masonry, the Tsai-Wu criterion [11] is proposed.

The Tsai-Wu criterion for plane stress conditions can be written in the form

$$
\begin{aligned}
& f_{m}\left(\sigma_{i j}\right)-F_{11} \sigma_{11}+F_{22} \sigma_{22}+F_{11} \sigma_{1111}^{2} \\
& +F_{22} \sigma_{2222}^{2}+F_{1212} \sigma_{12}^{2}+F_{1122} \sigma_{11} \sigma_{22}
\end{aligned}
$$

where $F_{i j}, F_{i j k l}$ are components of the second and fourth order anisotropic tensors, respectively. The criterion is usually identified as follows. Four of the model constants introduced are obtained from uniaxial compression and tension tests

$$
\begin{gathered}
F_{11}=\frac{1}{Y_{c}}-\frac{1}{Y_{t}}, \quad F_{1111}=\frac{1}{Y_{c} Y_{t}}, \\
F_{22}=\frac{1}{X_{c}}-\frac{1}{X_{t}}, \quad F_{2222}=\frac{1}{X_{c} X_{t}},
\end{gathered}
$$

where $Y_{c}, Y_{t}$ denote uniaxial compression and tension strength in $x_{1}$ direction (perpendicular to bed joints) and $X_{c}, X_{t}-$ in $x_{2}$ direction (parallel to bed joints), respectively. The value of $F_{1212}$ is typically obtained from result of pure shearing $X Y_{s}$ in $x_{1}-x_{2}$ plane

$$
F_{1212}=\frac{1}{X Y_{s}^{2}}
$$

Finally, the value of $F_{1122}$ component is obtained from result of equibiaxial tension $X Y_{t}$, as

$$
\begin{gathered}
F_{1122}=\frac{1}{2 X Y_{t}^{2}} \\
\cdot\left[1-X Y_{t}\left(F_{11}+F_{12}\right)-X Y_{t}^{2}\left(F_{1111}+F_{2222}\right)\right] .
\end{gathered}
$$

For the simplest loading configuration, $\alpha=0$, the $F_{1212}$ constant vanishes $\left(\sigma_{12}=0\right)$. The identification of the criterion for masonry microstructure has been then started with results obtained for that direction (Fig. 5). The results of uniaxial tension and compression tests in the $x_{2}$ direction can be taken directly from diagram as $X_{c}=1.52 \mathrm{MPa}$ and $X_{t}=0.56 \mathrm{MPa}$. Then by using formulas (10) the values of $F_{22}$ and $F_{2222}$ can be determined. As can be seen in Fig. 9, the values of uniaxial compression and tension in $x_{1}$ and the value of equibiaxial tension are affected by the previously described mechanism of localized shearing and have already been described by critical plane condition (8). Considering this, the values of $Y_{t}, Y_{c}$ and $X Y_{t}$ have been determined based on fitting the proposed criterion in results not influenced by shearing mechanism. Satisfactory results have been obtained using formulas (10) and (12) together with values $Y_{c}=1.43 \mathrm{MPa}$, $Y_{t}=0.7 \mathrm{MPa}$ and $X Y_{t}=0.43 \mathrm{MPa}$. In Fig. 14, conjunctions of identified Tsai-Wu model and critical plane criterion for direction $\alpha=0$ together with numerical results obtained previously for masonry microstructure are presented.

For identification of $F_{1212}$ value numerical results obtained for angle $\alpha=22.5^{\circ}$ have been utilized. The value of the constant has been determined also based on fitting the Tsai-Wu model in results not associated with localized shearing (Fig. 13). A very good agreement between identified model and numerical results has been obtained using value $X Y_{s}=0.28 \mathrm{MPa}$ together with formula (11). The identified conjunction of the criteria considered together with results of numerical analysis obtained previously for $22.5^{\circ}$ (Fig. 6) are presented in Fig. 15.

The identified criterion has been verified against numerical results obtained for different loading configuration than used previously for identification of the model. In Figs. 16 and 17, a comparison of identified model with results obtained for $\alpha=45^{\circ}$ and $\alpha=67.5^{\circ}$ is presented. The obtained agreement seem to be satisfactory, especially as in Fig. 16, where 
localized shearing is the governing mechanism of failure for almost all results.

In Figs. 18 and 19, the identified model has been compared with the results obtained numerically for directional uniaxial compression and tension tests (Figs. 3 and 4). It appears from the results presented that identified model is also in good agreement with these results.
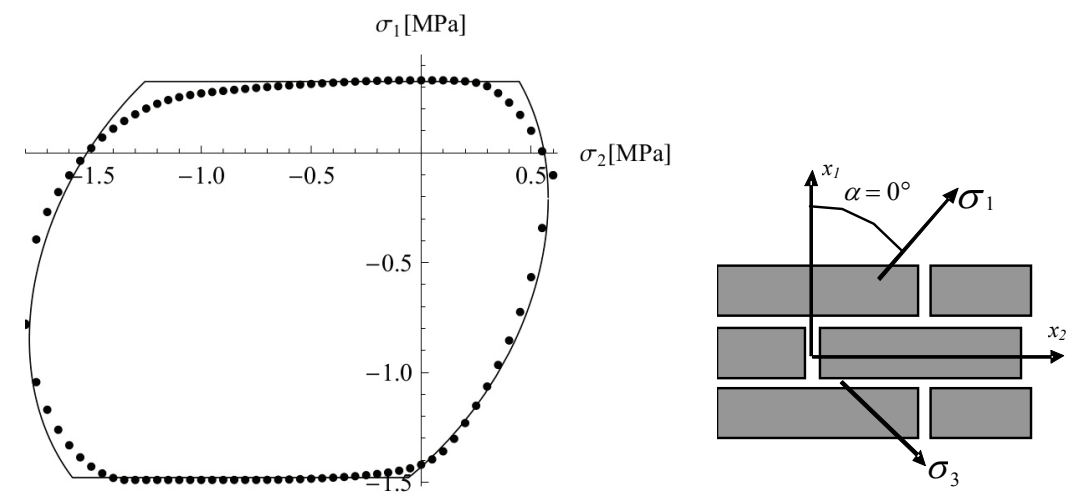

Fig. 14. Results from Fig. 5 (dots) together with identified model (line). Load angle $\alpha=0^{\circ}$
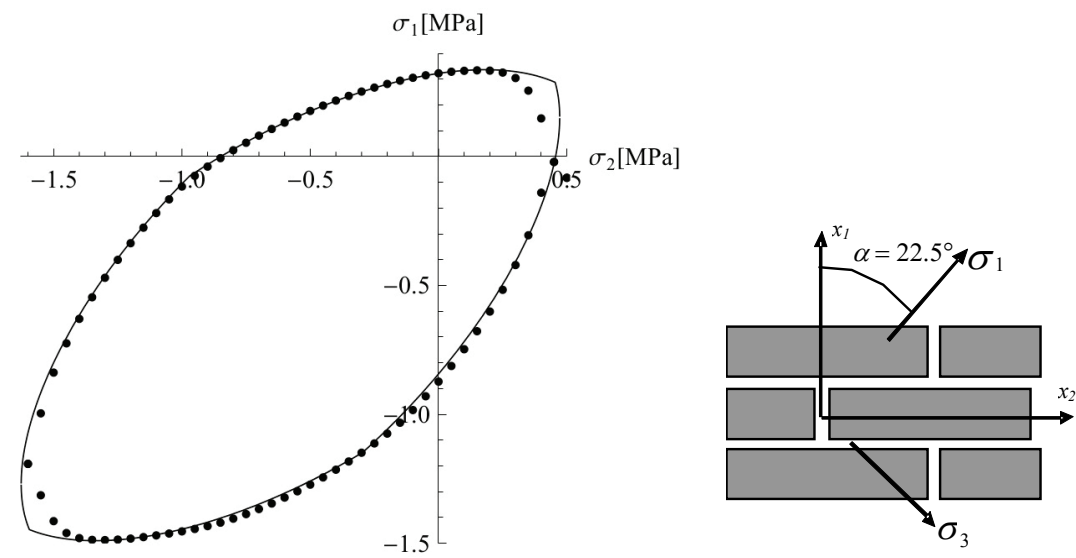

Fig. 15. Comparison of microstructural analysis results (dots) with identified model (line). Load angle $\alpha=22.5^{\circ}$
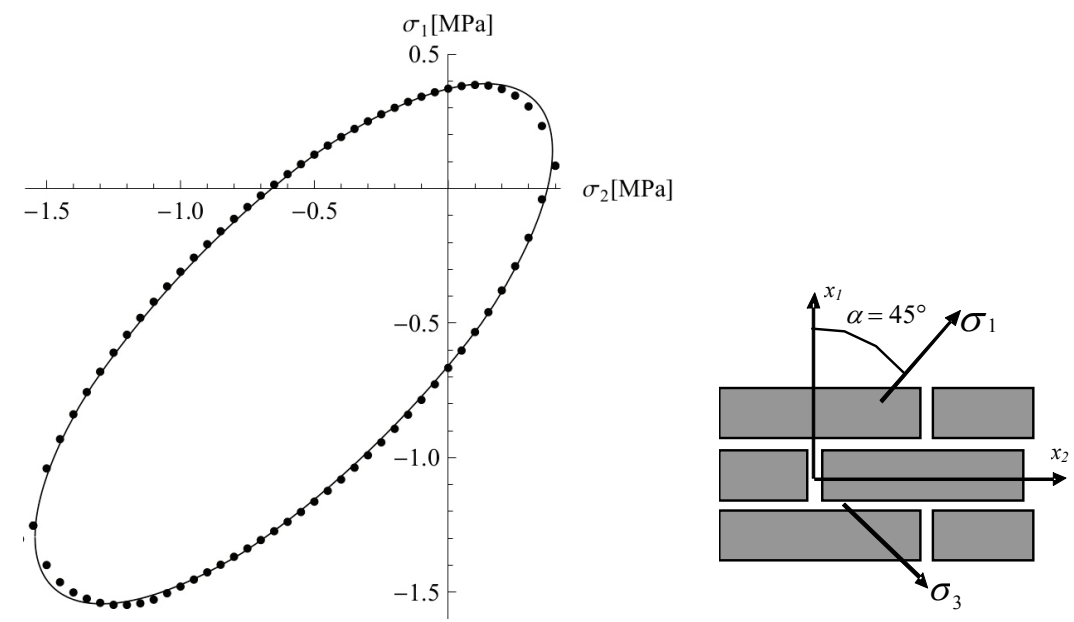

Fig. 16. Comparison of microstructural analysis results (dots) with identified model (line). Load angle $\alpha=45^{\circ}$ 

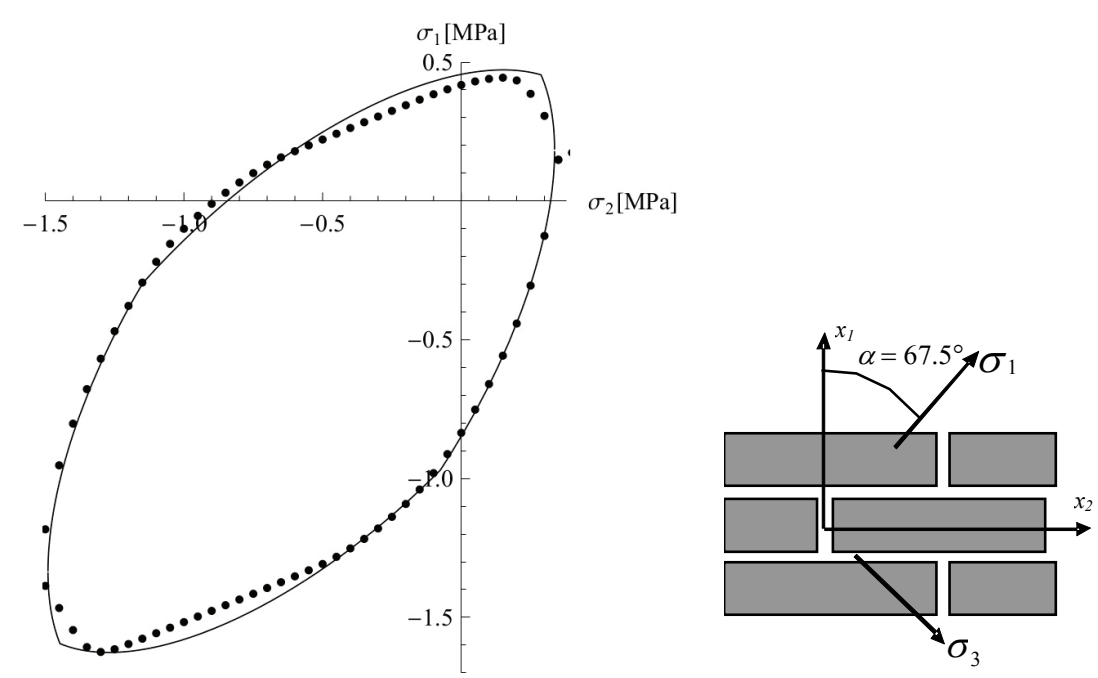

Fig. 17. Comparison of microstructural analysis results (dots) with identified model (line). Load angle $\alpha=67.5^{\circ}$
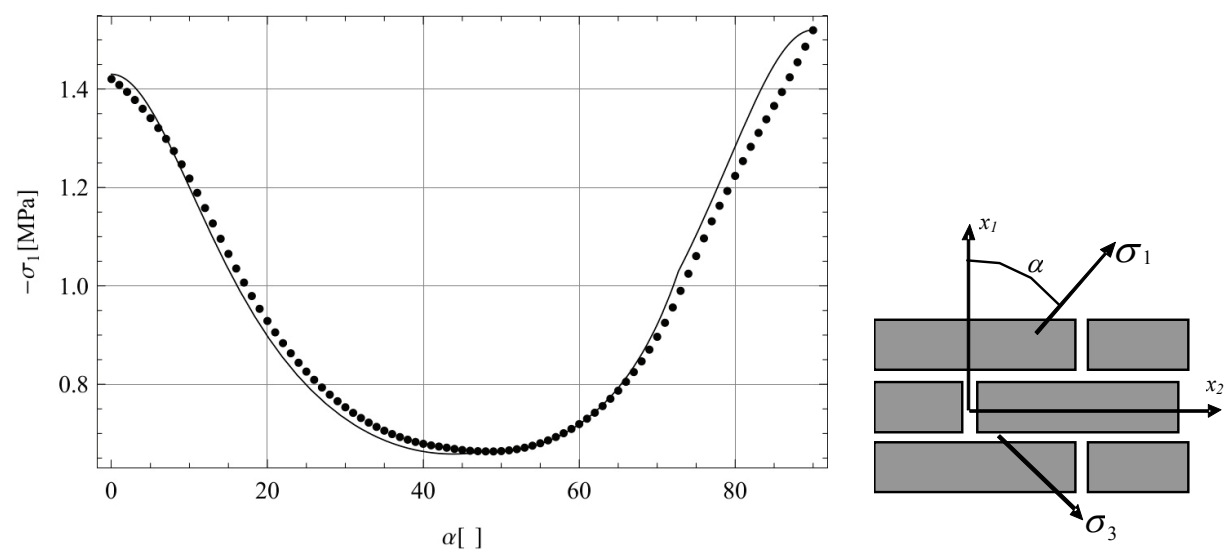

Fig. 18. Comparison of microstructural analysis (dots) with identified model (line). Directional compression test
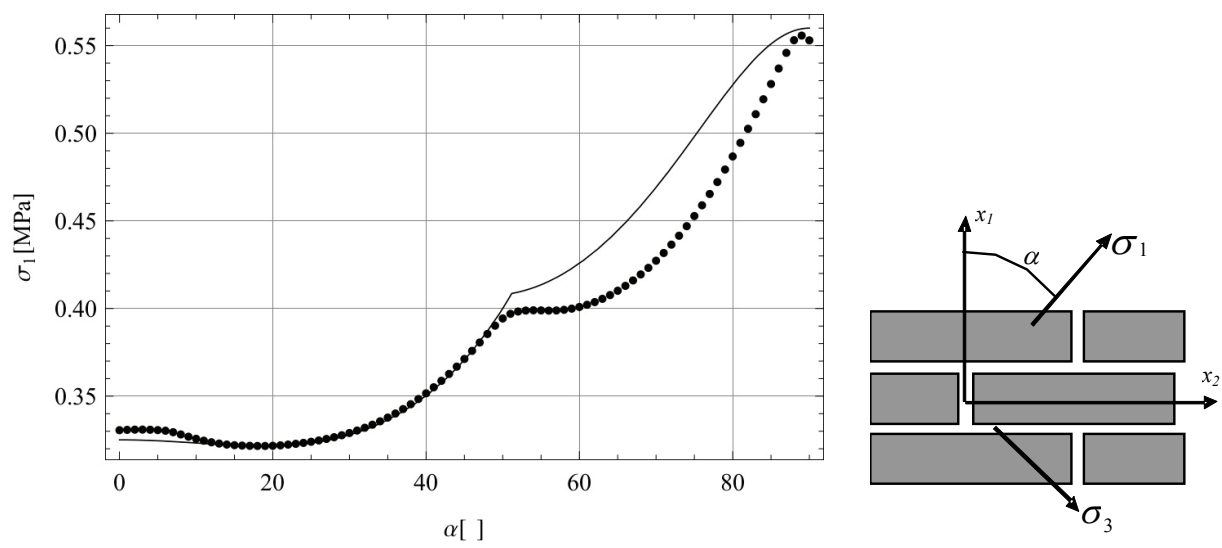

Fig. 19. Comparison of microstructural analysis (dots) with identified model (line). Directional tension test

\section{CONCLUSION}

In this work, a phenomenological strength criterion for in-plane loaded masonry has been proposed. The model has a form of conjunction of Jaeger [4] critical plane and Tsai-Wu [11] criteria. A procedure of identification of the proposed criterion has also been presented. The procedure has been based on fitting in results of lower bound strength assessment of masonry, obtained by micro-mechanics approach. The identified model has been verified against numerical results ob- 
tained for different loading paths. It appears from the diagrams presented that agreement between identified model and lower bound strength assessment for masonry microstructure is satisfactory.

The following conclusions can be drawn from the present study:

(i) The strength of masonry is bounded by strength of layered and block microstructures. For both of these microstructures exact solution (in the bound of numerical error limit) is easy to obtain. While these assessments seem to be very coarse, for many load paths they almost coincide, providing good estimation of masonry strength.

(ii) The mechanism of localized shearing along bed joints is a governing mechanism of the masonry microstructure failure for number of load paths. The resulting strength of the mechanism can be easily analytically described with critical plane criterion proposed by Jaeger [4].

(iii) It appears from the results presented that for all other more complicated mechanisms of masonry failure, when the Drucker-Prager criterion is assumed to be local strength condition for masonry components, the Tsai-Wu criterion provides satisfactory description of microstructure strength. This criterion can be easily identified using results of microstructural analysis for load paths not influenced by localized shearing in bed joints.

(iv) The conjunction of the two above conditions provides useful phenomenological model of masonry strength. Its simplicity and good agreement between identified model and numerical strength results obtained with micromechanics suggest that properly identified model can be widely used for estimation of strength in the modelling of large masonry structures

As has been mentioned before, the current study is a preliminary one. The above findings are subject to two main limitations:

(i) The local criterion used for constituents of masonry in this work is the Drucker-Prager one. It is known that this criterion (used without additional Cut-Off) strongly overestimates tensile strength of brickmortar interface and as a consequence also tensile strength of masonry microstructure. Adding Cut-Off at constituent level may result in the need of introducing additional part in phenomenological macroscopic description of masonry strength. The modified macroscopic criterion for brick masonry will be the subject of further studies. Also the approach presented is a simple in-plane limit analysis of microstructure and no 3D effects have been studied. In consequence, the obtained admissible macro stress field in the compression zone can be strongly underestimated. For these two reasons the results obtained so far are not realistic and thus no comparison with experimental data available in literature (see, for instance, Page [8]) has been provided. Further studies with updated model will include this comparison.

(ii) The micro-mechanic procedure utilized in this paper uses simple micro-stress fields constructed within periodic cell of masonry. The obtained lower bound assessment of masonry strength for some load paths can be inaccurate. The future study investigating more precise assessment, using, for instance, methodology proposed by Milani et al. [6] is needed.

\section{REFERENCES}

[1] AnthoIne A., Derivation of in-plane elastic characteristics of masonry through homogenization theory, International Journal of Solids and Structures, 1995, 32(2), 137-163, DOI: 10.1016/0020-7683(94)00140-R.

[2] De Buhan P., De Felice G., Homogenization approach to the ultimate strength of brick masonry, Journal of the Mechanics and Physics of Solids, 1997, 45(7), 1085-1104, DOI: 10.1016/S0022-5096(97)00002-1.

[3] De Felice G., Amorosi A., Malena M., Elasto-plastic analysis of block structures through a homogenization method, International Journal of Analytical and Numerical Methods in Geomechanics, 2010, 34(3), 221-247, DOI: 10.1002/nag.799.

[4] JAEGER J.C., Shear failure of anisotropic rocks, Geological Magazine, 1960, 97(1), 65-72.

[5] Kawa M., Pietruszczak S., Shieh-Beygi B., Limit states for brick masonry based on homogenization approach, International Journal of Solids and Structures, 2008, 45 (3-4), 998-1016, DOI: 10.1016/ j.ijsolstr.2007.09.015.

[6] Milani G., Lourenco P.B., Tralli A., Homogenised limit analysis of masonry walls, part I: failure surfaces; part II: structural examples, Computers and Structures, 2006, 84 (3-4), 166-195, DOI: 10.1016/j.compstruc.2005.09.005, 10.1016/ j.compstruc.2005.09.004.

[7] LydzBA D., KawA M., Failure mechanism of sedimentary rocks: micromechnics approach, In 2nd International Symposium On Computational Geomechanics (COMGEO II), Cavtat-Dubrovnik, 27-29 April, 2011, Rhodes, Greece: IC2E International Centre for Computational Engineering.

[8] PAGE A.W., The biaxial compressive strength of brick masonry, ICE Proceedings, 1981, 71(2), 893-906, DOI: 10.1680/iicep.1981.1825.

[9] Shieh-Beygi B., Pietruszczak S., Numerical analysis of structural masonry: mesoscale approach, Computers and Structures, 2008, 86, 21-22, 1958-1973, DOI: 10.1016/j.compstruc.2008.05.007.

[10] SUQUET P., Elements of homogenisation for inelastic solid mechanics, [in:] Homogenization Techniques for Composite Media, Lecture Notes in Physic, 1987, 272, 193-278, DOI: 10.1007/3-540-17616-0_15.

[11] TSAI S.W., Wu E.M., A general theory of strength for anisotropic materials, Journal of Composite Materials, 1971, 5(1), 58-80, DOI: 10.1177/002199837100500106. 